\title{
Research on Value Orientation of PE Teaching Material of Higher Traditional Chinese Medical University Based on the Method of Network Evaluation
}

\author{
Yali Xu, Kaihua Xu \\ School of Physical Education, Jiangxi University of Traditional Chinese Medicine, Nanchang, China \\ Police Training Management Department, Jiangxi People's Police College, Nanchang, China
}

Keywords: network evaluation; traditional Chinese medicine colleges; PE teaching materials; value orientation; model building

\begin{abstract}
The teaching material is the carrier of human knowledge and wisdom, and it must contain the value orientation of the direction of social development. However, at present the PE teaching material of higher traditional Chinese medical universities of our country has the value orientation of traditional structure and a little practice, and this situation runs counter to the requirement of education development in our country. At first, this paper expounds the concept of the method of network evaluation, and then it analyzes the advantage of using the method of network evaluation to evaluate the value orientation of PE teaching materials in higher traditional Chinese medical universities, finally it builds the evaluation model of value orientation that based on the method of network evaluation, and it can use this model to have researches and analysis on the value orientation of PE teaching materials in higher traditional Chinese medical universities. And it can conclude that the value orientation of the PE teaching material in higher traditional Chinese medical universities should be oriented to the modernization and at the same time it should meet characteristics of education development.
\end{abstract}

\section{Introduction}

Since reform and opening-up, the education of higher traditional Chinese medical universities in our country has made great progress, and it has trained a batch of modern talents for the country and society [1]. According to the related information and data of the higher traditional Chinese medical universities, it can make scientific and reasonable evaluation of the current education mode and the selection of teaching material, at the same time it can provide guidance to the selection of value orientation of the PE teaching materials in higher traditional Chinese medical universities. In the paper of the research on the several issues of development of higher education of Chinese medicine at the beginning of the 21st century - the quality evaluation and development strategy of value orientation, Tianren Yang pointed out that China's higher medical education was in the key period of reform and development, and it was experiencing the important stage of reselecting the value orientation, and the transformation of education must face the modernization and conform to the requirements of the time[2,3].

\section{The concept and characteristic of the method of network evaluation}

\section{The Concept of the Network Evaluation}

The method of network evaluation that based on the computer system can break the time and geographical constraints of the method of traditional evaluation, and it also can use the Internet to have remote control of the whole process of evaluation[4]. And the structure of the network evaluation system is shown in Figure 1.

It can be seen from Figure 1 that the network evaluation system mainly includes users, reading system of computer information, information processing and terminal of result evaluation [5]. The reading system of computer information and information processing and terminal of result evaluation 
are the key parts of the network evaluation system, and the reading and processing systems of information are shown in Figure 2.

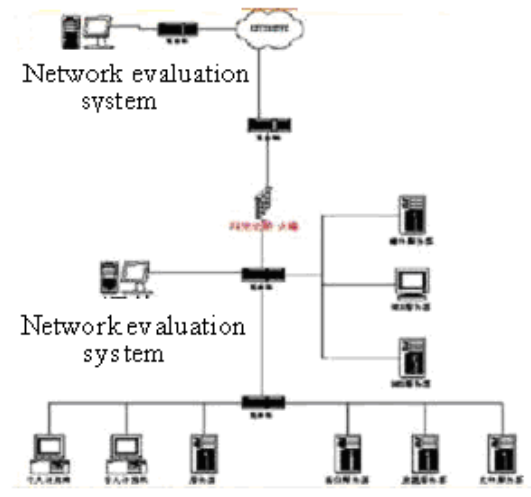

Figure 1. The structure of the network evaluation system

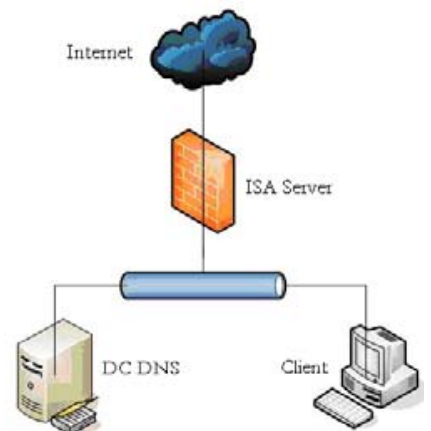

Figure 2. Reading and processing systems of computer information

\section{The Characteristics of the Network Evaluation}

The method of network evaluation is different from the traditional evaluation methods, and it breaks the limitation of time and space, and its main characteristics are the following several aspects [6,7]:

The main body of evaluation is in a popular style.

The variety of evaluation modes.

The evaluation technique is modern.

The analysis of the advantage of network evaluation's application in the value orientation of PE teaching materials in higher traditional Chinese medical universities

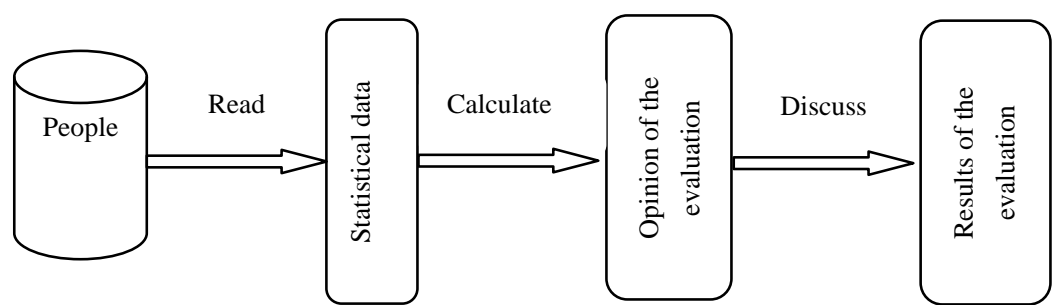

Figure 3. Flow chart of the mode of traditional evaluation

In Figure 3, the mode of traditional assessment mainly uses the main body of assessment to read the statistical data, and then it calculates the data and obtains the opinion of the evaluation, and finally it has the discussion and votes the consistent evaluation results [8-10]. The entire process is time-consuming and very trouble, and the evaluation object also has significant limitations. Due to the man-made causes, evaluation results are often not accurate.

Then it introduces the method of network evaluation that based on the computer technology. In the process of this evaluation, firstly the main body inputs the statistical data, and then the computer system has data processing and obtains the evaluation results, and the structure of evaluation network is shown in Figure 4. 


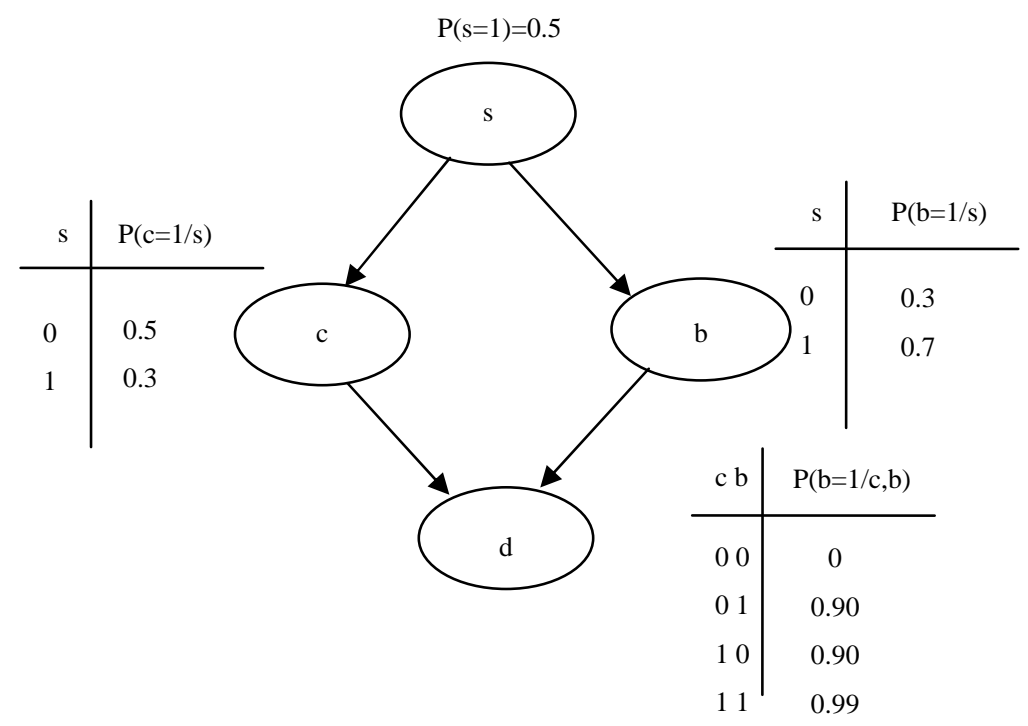

Figure 4. Structure diagram of evaluation network

When the statistical data and the weight coefficient are input into the computer and according to the Bayesian formula, the computer can carry on the operations. It can be assumed that its weight coefficient is 1 , and then the calculation process is as follows:

$$
\begin{gathered}
P(d=1)=\sum_{s, c, b} P(s, c, b, d)=\sum_{c, b} P(d=1 \mid c, b) \sum_{s} P(c, b \mid s) P(S)= \\
\sum_{c, b} P(d=1 \mid c, b)[p(c, b \mid s=1) p(s=1)+p(c, b \mid s=0) p(s=0)]= \\
\mathrm{P}(\mathrm{d}=1 \mid \mathrm{c}=1, \mathrm{~b}=1)[\mathrm{P}(\mathrm{c}=1, \mathrm{~b}=1 \mid \mathrm{s}=1) \cdot \mathrm{P}(\mathrm{s}=1)+\mathrm{P}(\mathrm{c}=1, \mathrm{~b}=1 \mid \mathrm{s}=0) \\
\mathrm{P}(\mathrm{s}=0)]+\mathrm{P}(\mathrm{d}=1 \mid \mathrm{c}=1, \mathrm{~b}=0)[\mathrm{P}(\mathrm{c}=1, \mathrm{~b}=0 \mid \mathrm{s}=1) \cdot \mathrm{P}(\mathrm{s}=1)+\mathrm{P}(\mathrm{c}=1, \\
\mathrm{b}=0 \mid \mathrm{s}=0) \mathrm{P}(\mathrm{s}=0)]+\mathrm{P}(\mathrm{d}=1 \mid \mathrm{c}=0, \mathrm{~b}=1)[\mathrm{P}(\mathrm{c}=0, \mathrm{~b}=1 \mid \mathrm{s}=1) \cdot \mathrm{P}(\mathrm{s}=1) \\
+\mathrm{P}(\mathrm{c}=0, \mathrm{~b}=1 \mid \mathrm{s}=0) \mathrm{P}(\mathrm{s}=0)]+\mathrm{P}(\mathrm{d}=1 \mid \mathrm{c}=0, \mathrm{~b}=0)[\mathrm{P}(\mathrm{c}=0, \mathrm{~b}=0 \mid \mathrm{s}=1) \cdot \\
\mathrm{P}(\mathrm{s}=1)+\mathrm{P}(\mathrm{c}=0, \mathrm{~b}=0 \mid \mathrm{s}=0) \mathrm{P}(\mathrm{s}=0)]
\end{gathered}
$$$$
\mathrm{P}(\mathrm{s}=0)]+\mathrm{P}(\mathrm{d}=1 \mid \mathrm{c}=1, \mathrm{~b}=0)[\mathrm{P}(\mathrm{c}=1, \mathrm{~b}=0 \mid \mathrm{s}=1) \cdot \mathrm{P}(\mathrm{s}=1)+\mathrm{P}(\mathrm{c}=1 \text {, }
$$$$
+\mathrm{P}(\mathrm{c}=0, \mathrm{~b}=1 \mid \mathrm{s}=0) \mathrm{P}(\mathrm{s}=0)]+\mathrm{P}(\mathrm{d}=1 \mid \mathrm{c}=0, \mathrm{~b}=0)[\mathrm{P}(\mathrm{c}=0, \mathrm{~b}=0 \mid \mathrm{s}=1) \text {. }
$$

After the calculation, the internal program of the computer can automatically eliminate incorrect evaluation results, and at the same time the correct evaluation results have two-way derivation to ensure the correctness and the rationality of the evaluation results. This evaluation process is simple and quick, and it saves a lot of time, and it has less cost of manpower and material resources. The evaluation objects can be anything, and evaluation results completely rules out the influence of human, so it is much better than the traditional evaluation method [11]. Therefore, it meets the characteristics of times and the requirements of education to use the method of network evaluation to choose the value orientation of the PE teaching materials in higher traditional Chinese medical universities.

\section{Model building of value orientation of the PE teaching materials that based on the network evaluation}

If we want to build the model of value orientation of the PE teaching materials in higher traditional Chinese medical universities that based on the network evaluation, firstly the network evaluation system that meets the teaching characteristic and teaching requirement of the higher traditional Chinese medical universities should be set up, and it is shown in the Figure 5.

$\mathrm{PE}$ teaching materials in higher traditional Chinese medical universities

The network evaluation system of value orientation of the PE teaching materials in higher traditional Chinese medical universities is a network platform, and it can evaluate that the value orientation is correct or not. The users can complete the evaluation by themselves, and it also can use Internet to let the public participate in the assessment and give their views.

The second step is the input and processing of the data and the structure of data and information processing is shown in Figure 6. 


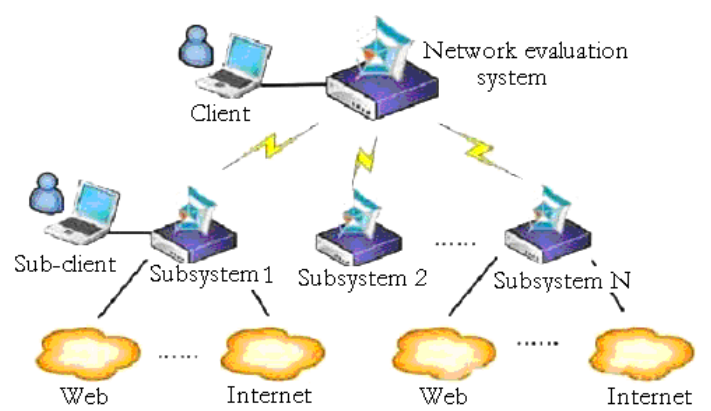

Figure 5. Network evaluation system of value orientation of the

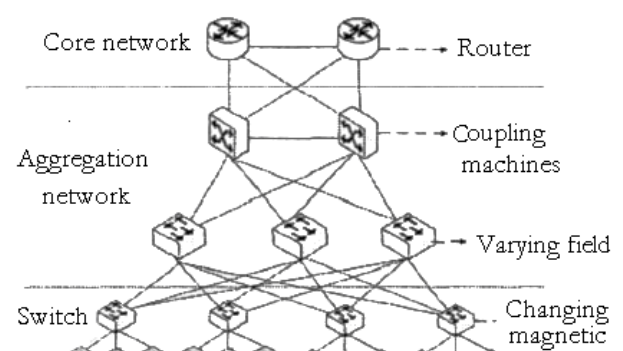

000 ren

Figure 6. Structure diagram of data and information processing of network evaluation system

The structure of data and information processing of network evaluation system shows the input, transformation, process of the data. This process only needs the user to input relevant data and information of sports teaching in higher traditional Chinese medical universities into the computer, and the network evaluation system considers the situation that the large amount of data can not be input into the computer once. So it designs the perceived TCP protocol procedures, and it can make the user input data repeatedly without affecting the evaluation process and the protocol program is shown as follows:

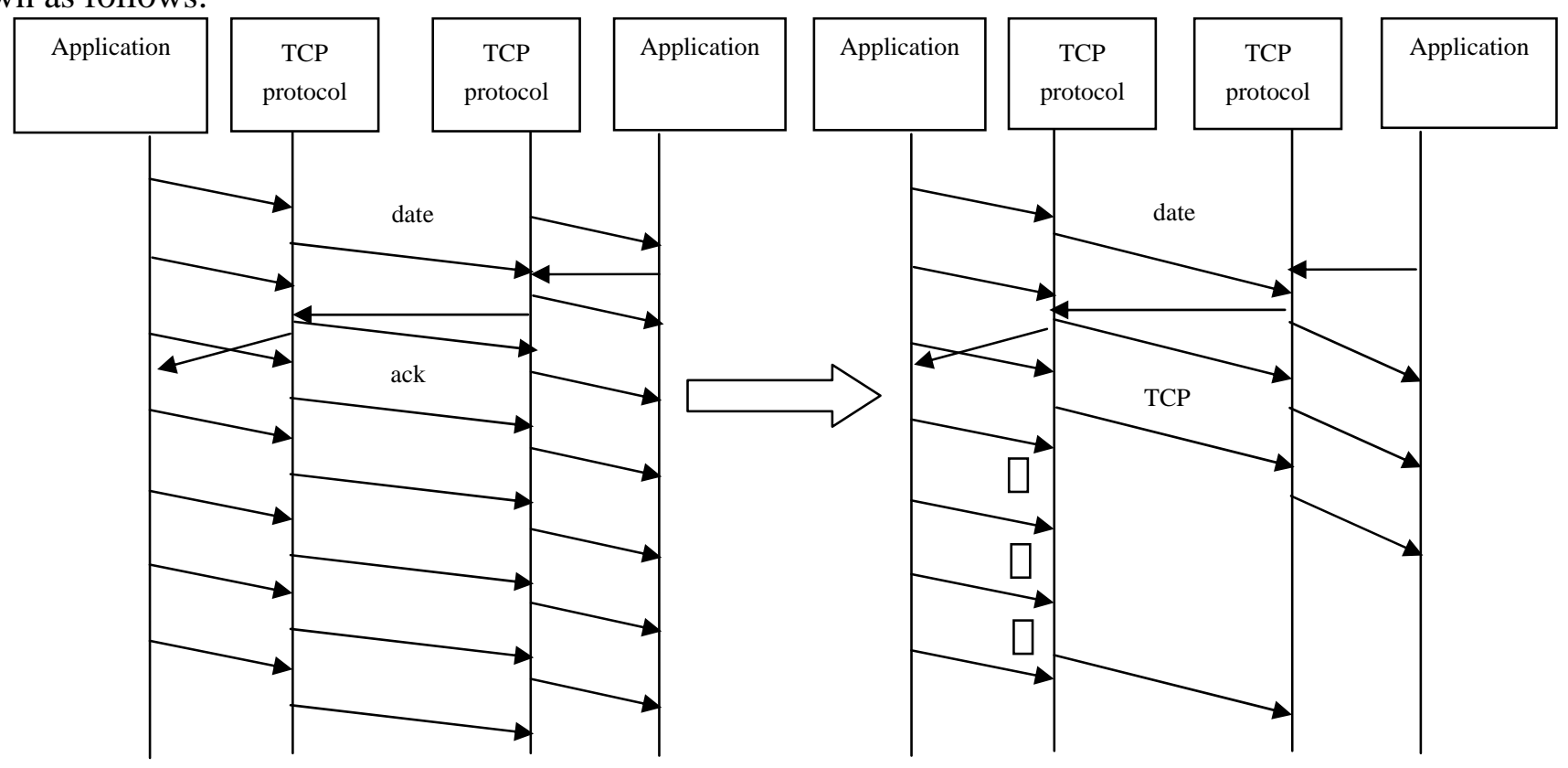

Figure 7. Sketch map of program of TCP protocol

The conversion and processing of data and information of network evaluation system is mainly the process of gradient computation. Its operational formula is $\nabla \eta(\theta)=\sum_{i \in \Phi} \pi_{i}(\theta) \cdot\left(\nabla r_{i}(\theta)+\sum_{j \in \Phi}\left(a_{i j}(\theta) \cdot L_{i j}(\theta) g_{j}(\theta)\right)\right)$, and the gradient cycle of the $\mathrm{n}$ statistical data is $t_{m}<n \leq t_{m+1}$, and it can be calculated out by the $\bar{g}_{i_{n}}(\theta, \bar{\eta})=\sum_{k=n}^{t_{m+1}-1}\left(r_{i_{k}}(\theta)-\bar{\eta}\right)$. When it has the input errors due to human factors, and the data is clearly not the same with other gradient data, and then the system will automatically correct the data, and there has $\bar{g}_{i_{n}}(\theta, \bar{\eta})=0$. When there has $n=t_{m}$, it can be calculated through the formula $\overline{\nabla \eta_{m}}(\theta, \bar{\eta})=\sum_{n=t_{m}}^{t_{m+1}-1} \bar{g}_{i_{n}}(\theta, \bar{\eta}) L_{i_{n-1} i_{n}}(\theta)+\nabla r_{i}(\theta)$.

Using the method of network evaluation to assess the value orientation of the PE teaching materials in higher traditional Chinese medical universities can save a lot of manpower and material resources, the whole process are completed by computer system, and it can avoid the influence of 
man-made factors on the assessment results, and the humanized program design makes the evaluation results meet the requirement of sports teaching in the higher traditional Chinese medical universities.

\section{Conclusion}

In our country, higher education cultivated a large number of modern knowledge-based talents, and it made great contribution to enhance the overall national strength. Therefore, our country never relaxed the attention of the higher education. According to the problems of the unclear and inaccurate value orientation of institutions of higher learning in the process of rapid development of education, the network evaluation method that based on computer systems is proposed in this paper. And compared with the traditional evaluation methods, this method can save time and labor, and the evaluation process can effectively avoid artificial factors, so the evaluation result is very accurate. In conclusion, this research can provide some help for the development of sports education in the higher traditional Chinese medical universities.

\section{Acknowledgment}

This work was financially supported by the Jiangxi Province Provincial Teaching Reform Foundation Item (JXJG-06-8-36), its project name is the higher Chinese medicine universities sports teaching value orientation research in twenty-first Century.

\section{References}

[1] Xingeng Liu, Guanping Cao, Bangjie Liu. Methodology of education network of ideological and political evaluation[J]. Research of ideological and political education, 2011(06): 36-39

[2] Tianren Yang. Research on the several issues of development of higher education of Chinese medicine at the beginning of the 21st century research-the quality evaluation and development strategy of value orientation[J]. Journal of Heilongjiang university of Chinese medicine, 2010(11): 59-62.

[3] Hua Wang. The thought of development strategy of our country's education of higher traditional Chinese medicine [J]. Journal of Hubei college of traditional Chinese medicine, 2011(05):24-27.

[4] Xiaowei Yin, Wenxue Qian, Liyang Xie. Method of Bayesian network assessment of system reliability [J]. Journal of aviation, 2012 (08): 55-58.

[5] Linrun Qiu. Analysis and design of network evaluation system of ideological and political evaluation courses in colleges and universities [J]. Computer CD software and applications, 2012 (02): 67-70.

[6] Tang Xu, Qinghuan Zhou, Shan Wang. Analysis of network evaluation results of teaching quality of the clinical medicine theory [J]. Journal of medical education, 2011(05):28-31

[7] Yu Gao, Yunfei Ma. EGPRS network assessment and optimization based on user perception[J]. Journal of network gauge and network optimal column, 2010(12): 72-75.

[8] Qiupu Liu. Thought of scientific evaluation of moral education in colleges and universities[J]. Journal of Wuhan university of technology (social science edition), 2011(06):66-69.

[9] F. Yu, V. Krishnamurthy, and V. C. M. Leung. Cross layer optimal connection admission control for variable bit rate multimedia traffic in packet wireless CDMA networks[J]. IEEE Trans. Signal Processing, 2010(09): 80-83.

[10]R. Bolla, R_ Bruschi, K. Christensen, F Cucchietti, F. Davoli, and S. Singh. The Potential Impact of Green Technologies in Next Generation Wireline Networks - Is There Room for Energy Savings Optimization [J]. IEEE Commun, 2011(02):32-35.

[11]B.Nordman, K.Christensen.Jul. Reducing the Energy Consumption of Network Devices [S]..IEEE 802.3 Tutorial, 2011(09):66-68. 